\title{
PARTO PRETÉRMINO Y SUFRIMIENTO FETAL SECUNDARIOS A PERITONITIS POR APENDICITIS AGUDA PERFORADA
}

\author{
Juan José Hidalgo M. ${ }^{1}$, Marta Molina P. ${ }^{1}$, Begoña Varo GM. ${ }^{1}$, Salvador Rivas R. ${ }^{2}$, \\ José Ramón Bernabeu A. ${ }^{3}$, Alfredo Perales M. ${ }^{1}$
}

\footnotetext{
${ }^{1}$ Servicio de Obstetricia, ${ }^{2}$ Servicio de Anatomía Patológica, ${ }^{3}$ Servicio de Ginecología, Hospital Maternal Universitario La Fe, Valencia, España.
}

\section{RESUMEN}

La apendicitis aguda es la urgencia quirúrgica no obstétrica más frecuente durante el embarazo. Se ha asociado a parto pretérmino y a morbimortalidad fetal y materna, especialmente cuando se complica con peritonitis. Los cambios anatómicos, fisiológicos y bioquímicos que se producen durante la gestación pueden alterar los síntomas y signos típicos asociados a la apendicitis. Esto puede retrasar el diagnóstico y dar lugar a un aumento de la morbimortalidad materna y fetal. Presentamos el caso de una paciente gestante de 35 semanas en que la dificultad en el diagnóstico de apendicitis aguda conllevó a un cuadro de peritonitis, secundario a perforación apendicular, que causó dinámica uterina prematura y sufrimiento fetal.

\section{PALABRAS CLAVE: Apendicitis, embarazo, parto prematuro, sufrimiento fetal}

\section{SUMMARY}

Acute appendicitis is the most common non-obstetric surgical emergency in pregnancy. It has been associated with premature labour and fetal and maternal morbidity and mortality, especially when complicated with peritonitis. Anatomical, physiological and biochemical changes during pregnancy may alter typical symptoms and signs associated with appendicitis. This can result in delayed diagnosis and lead to an increase in mother's and fetus morbi-mortality. We present a case of a pregnant woman at 35 weeks of gestation in whom the difficulty in diagnosis of acute appendicitis resulted in the appearance of peritonitis because of appendix perforation, which was the cause of preterm labour and fetal distress.

\section{KEY WORDS: Appendicitis, pregnancy, premature labor, fetal distress}

\section{INTRODUCCIÓN}

La incidencia de abdomen agudo durante la gestación es de 1/500-635 embarazos. Su diagnóstico en ocasiones es difícil debido a que el útero grávido desplaza otros órganos intraabdominales, lo que dificulta la exploración física. Además, sus principales síntomas como son, dolor abdominal, náuseas y vómitos, muestran una alta prevalencia en las pacientes obstétricas normales (1). De las diferentes causas de abdomen agudo, la apendicitis es la más frecuente en las mujeres gestantes, con una incidencia de 0,15-2,10/1000 embarazos, produciéndose usualmente en el segundo y tercer 
trimestre de la gestación.

La apendicitis aguda muestra una incidencia similar en mujeres gestantes y no gestantes, aunque con una frecuencia de perforación más alta en las primeras. La mortalidad materna es muy baja en los casos de apendicitis no complicada, pero puede alcanzar el $4 \%$ en casos de gestación avanzada y perforación. La mortalidad fetal oscila entre el 0$1,5 \%$ en casos de apendicitis simple, pero puede aumentar hasta el $20-35 \%$ en aquellos en los que se asocia perforación (2,3). Son pocos los casos publicados en la bibliografía médica en los últimos 20 años en los que la perforación de un proceso apendicular desencadene el inicio de dinámica uterina prematura y sufrimiento fetal grave (4-7).

El objetivo de esta comunicación es la presentación de un caso de perforación apendicular y peritonitis subsiguiente, que conllevaron a un cuadro de dinámica uterina prematura y sufrimiento fetal, que obligó a la interrupción del embarazo mediante cesárea de urgencia.

\section{Caso clínico}

Paciente de 42 años, con antecedentes de hipertensión arterial, diabetes mellitus tipo 1 en tratamiento insulínico, hipercolesterolemia y obesidad, que cursaba la semana $35+6$ de su sexta gestación (cuatro partos y un aborto en los embarazos anteriores), que consultó en el Servicio de Urgencias del Hospital Maternal Universitario, Valencia, España, por presentar cuadro de malestar general, náuseas y vómitos de repetición, sensación distérmica y dolor en hemiabdomen inferior y en región lumbar. Durante los 7 días previos, la paciente consultó en dos ocasiones por síntomas similares de menor intensidad. Tras exploración física, análisis sanguíneo, ecografía y cardiotocograma dentro de la normalidad, los síntomas fueron atribuidos a infección urinaria y emesis gravídica, siendo tratados farmacológicamente de forma ambulatoria.

En el momento de la consulta actual, la paciente presentaba temperatura de $37,5^{\circ} \mathrm{C}$, exploración física con cérvix uterino de consistencia dura, permeable a un dedo y de $2 \mathrm{~cm}$ de longitud, con parámetros analíticos normales salvo por la presencia de 13.600 leucocitos $/ \mathrm{mm}^{3}$ (85,8\% de neutrófilos) y proteína C-reactiva de 153,8 mg/l. No había evidencia de rotura de membranas. En la ecografía se observó un feto vivo en presentación cefálica, con biometría acorde a la edad gestacional, placenta en cara anterior normalmente inserta y líquido amniótico en cantidad normal. El registro cardiotocográfico mostró un patrón fetal con variabilidad mínima y presencia de deceleraciones tardías repetidas en la frecuencia cardiaca, así como taquisistolia en el patrón de dinámica uterina (Figura 1).

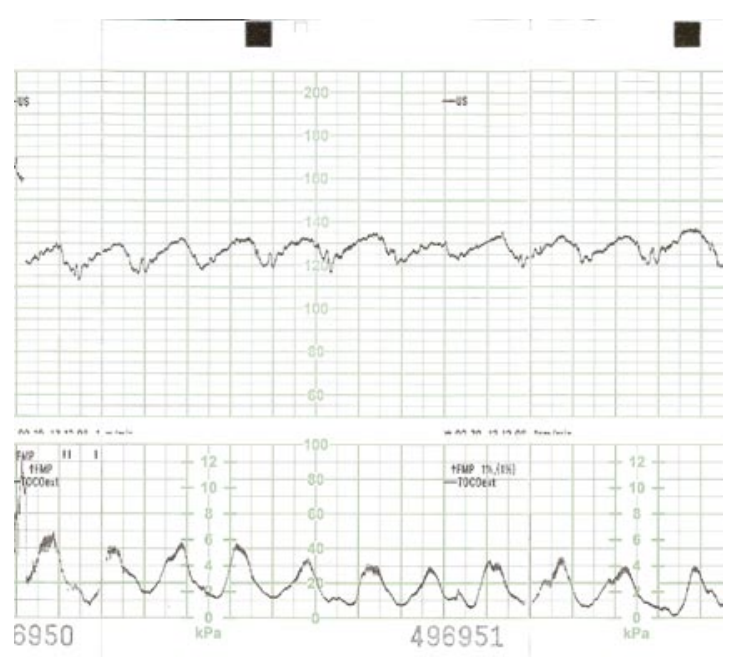

Figura 1. Cardiotocografía. Se observa variabilidad disminuida y desaceleraciones tardías de la frecuencia cardiaca fetal, y taquisistolia en la dinámica uterina.

Debido a la sospecha de pérdida de bienestar fetal, se indicó la realización de una cesárea urgente. En el momento de la apertura del peritoneo, se observó la existencia de abundante líquido ascítico purulento, así como un apéndice de aspecto gangrenoso. El estudio anatomopatológico posterior informó de apéndice cecal congestivo con depósitos purulentos en la serosa (Figura 2). El plastrón periapendicular fue extirpado una vez finalizada la extracción fetal y el cierre uterino. Se obtiene recién nacido masculino de $2.950 \mathrm{~g}$, con puntuaciones de 4 y 8 en el test de Apgar, $\mathrm{pH}$ arterial de 6,63 y venoso de 6,70, que inicialmente requirió únicamente reanimación con oxígeno a través de mascarilla y posteriormente intubación e ingreso en $\mathrm{UCI}$ neonatal durante 8 horas, por respiración irregular e hipotonía. Permanece ingresado durante 5 días en la Unidad de Reanimación con mejoría progresiva de su situación clínica. Madre e hijo fueron dados de alta 7 días después del parto en buenas condiciones.

\section{DISCUSIÓN}

La dificultad para realizar el diagnóstico precoz de apendicitis en la gestante puede conllevar un retraso en el tratamiento quirúrgico y la aparición de complicaciones tanto maternas como fetales. El desencadenamiento de dinámica uterina prematura 
y el parto pretérmino son algunas de estas complicaciones de la apendicitis durante el embarazo, pudiendo alcanzar el $83 \%$ y el $13 \%$, respectivamente durante el tercer trimestre $(3,8)$.

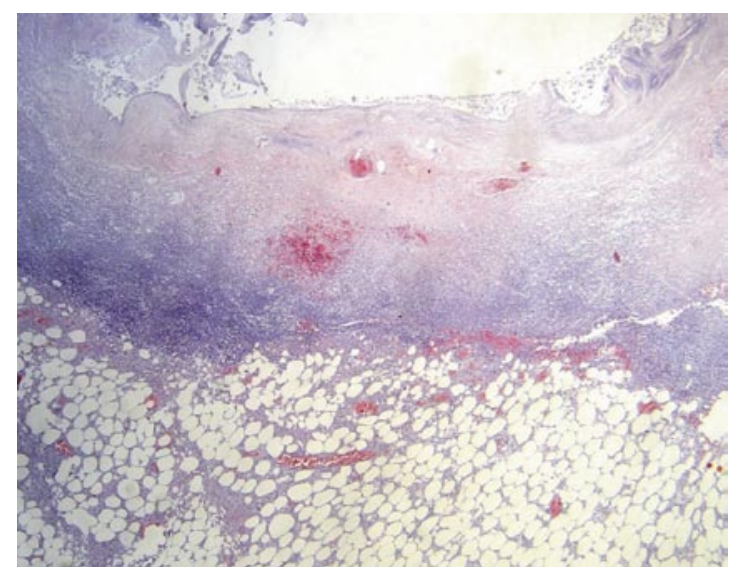

Figura 2. Corte histológico de apéndice. Pared apendicular necrótica y gangrenosa. Infiltrado de polimorfonucleares en el tejido adiposo del meso con presencia de vasos sanguíneos congestivos.

El aumento del tono uterino podría derivarse del inicio de procesos inflamatorios en su proximidad, con el aumento de la secreción de numerosos mediadores inflamatorios como la IL-1, 6, 8 y 10 o el TNF- $\alpha$, lo que podría desencadenar el inicio del parto y el deterioro del bienestar fetal (4). Además, el útero grávido puede constituirse en la pared medial de un absceso apendicular o de una peritonitis incipiente, por lo que resultaría inmediatamente afectado por el proceso inflamatorio e infeccioso. El efecto resultante para el útero sería la aparición de contracciones por un mecanismo de tipo irritativo (9). Es lógico pensar que en los casos en que la perforación apendicular complica el cuadro inicial, los procesos descritos aumentan su intensidad y consecuencias, más si en muchos casos, la perforación se acompaña de una infección peritoneal grave. El riesgo de perforación es mayor en las mujeres gestantes (43\% vs. $19 \%$ en la población general) y aumenta con la edad gestacional, siendo el mayor predictor de mortalidad en esta patología (10).

El caso que describimos puede considerarse como un claro ejemplo de lo expuesto. El retraso en el diagnóstico del proceso inflamatorio apendicular, dado lo dificultoso de su reconocimiento clínico en una mujer con gestación avanzada, originó el desarrollo de la perforación del apéndice y la peritonitis consiguiente. Como consecuencia, la afectación uterina dio inicio a una intensa dinámica uterina y a un cuadro de deterioro fetal grave que afortunadamente se resolvió con la realización de una cesárea urgente. Respecto a esto, en algunos trabajos se ha señalado, una relación directa entre el intervalo del comienzo de los síntomas y el momento de la intervención, con la incidencia de perforación apendicular y el aumento de la morbilidad perinatal (7), lo cual queda reflejado asimismo en la evolución clínica y diagnóstica que mostró nuestra paciente.

Los síntomas más comunes de la apendicitis son el dolor abdominal, más frecuente en el cuadrante inferior derecho, el dolor de rebote y la defensa a la exploración. Éstos son menos frecuentes en la gestación avanzada debido a la laxitud de los músculos de la pared abdominal y a la interposición del útero entre el apéndice y la pared abdominal anterior. Además, el crecimiento del útero grávido puede desplazar el apéndice a superior y lateralmente, lo cual puede resultar en dolor en flanco o en región lumbar, lo que con frecuencia es confundido, tal y como ocurrió con una de las visitas anteriores de nuestra paciente, con infección del tracto urinario o pielonefritis.

La fiebre no ha sido demostrada como un signo fiable de apendicitis, al igual que los hallazgos de laboratorio, incluyendo leucocitosis y proteína C-reactiva, ya que pueden verse alterados fisiológicamente durante el embarazo. La ecografía puede ayudar al diagnóstico sobre todo en el primer trimestre, pero resulta menos útil en la gestación avanzada, sobre todo en el tercer trimestre, por la difícil visualización del apéndice por el útero grávido $(1,3,10)$. Por todo ello, en la mujer gestante, una correcta historia clínica y la exploración física, junto con un alto índice de sospecha, son los mejores indicadores para realizar el diagnóstico de apendicitis.

\section{CONCLUSIÓN}

Este caso es ilustrativo de que la apendicitis debe ser considerada en el diagnóstico diferencial en la gestante con dolor abdominal de reciente inicio. El dolor en el cuadrante inferior derecho, la anamnesis minuciosa y una detallada exploración física, son los mejores indicadores para realizar el diagnóstico. Resulta urgente hacer el diagnóstico correcto, dado que se trata de un proceso de alto riesgo de morbimortalidad materno-fetal. La dificultad del diagnóstico se debe a los cambios anatómicos, fisiológicos y bioquímicos, propios del embarazo, que puede resultar en el retraso diagnóstico. La intervención temprana es decisiva para evitar o reducir las complicaciones. 


\section{BIBLIOGRAFÍA}

1. Augustin G, Majerovic M. Non-obstetrical acute abdomen during pregnancy. Eur J Obstet Gynecol Reprod Biol 2007;131:4-12.

2. Humes DJ, Simpson J. Acute appendicitis. BMJ 2006;333:530-4.

3. Guttman R, Goldman RD, Koren G. Appendicitis during pregnancy. Can Fam Physician 2004;50:355-7.

4. Lehner R, Tringler B, Stengg K, Goharkhay N. Premature labor in a women with perforating appendicitis at 36 weeks of gestation. J Reprod Med 2002;47:327-8.

5. Somani RA, Kaban G, Cuddington G, McArthur R. Appendicitis in pregnancy: a rare presentation. CMAJ 2003;168:1020.
6. Auguste T, Murphy B, Oyelese Y. Appendicitis in pregnancy masquerading as recurrent preterm labor. Int $\mathrm{J}$ Gynecol Obstet 2002;76:181-2.

7. Zhang Y, Zhao YY, Qiao J, Ye RH. Diagnosis of appendicitis during pregnancy and perinatal outcome in the late pregnancy. Chin Med J 2009;122:521-4.

8. Mourad J, Elliott JP, Erickson L, Lisboa L. Appendicitis in pregnancy: New information that contradicts long-helds clinical beliefs. Am J Obstet Gynecol 2000;182:1027-9.

9. Wright EA. A case of acute appendicitis in pregnancy. Aust NZ J Obstet Gynaecol 1987;27:256-7.

10. Borst AR. Acute appendicitis: Pregnancy complicates this diagnosis. JAAPA 2007;20:36-41. 